\title{
Failure to clear intra-monocyte HIV infection linked to persistent neuropsychological testing impairment after first-line combined antiretroviral therapy
}

\author{
B. Shiramizu, \\ Hawaii Center for AIDS, University of Hawaii-Manoa, Honolulu, HI, USA; Department of \\ Pediatrics, University of Hawaii-Manoa, Honolulu, HI, USA; Department of Medicine, University of \\ Hawaii-Manoa, Honolulu, HI, USA; Hawaii John A. Burns School of Medicine, Hawaii Center for \\ AIDS, 651 llalo St BSB 325A, Honolulu, HI 96813, USA

\section{J. Ananworanich,} \\ Hawaii Center for AIDS, University of Hawaii-Manoa, Honolulu, HI, USA; Southeast Asia \\ Research Collaboration with Hawaii (SEARCH), The Thai Red Cross AIDS Research Centre, \\ Bangkok, Thailand; Faculty of Medicine, Chulalongkorn Hospital, Bangkok, Thailand; HIV \\ Netherlands Australia Thailand Research Collaboration, The Thai Red Cross AIDS Research \\ Centre, Bangkok, Thailand
}

\section{T. Chalermchai,}

Southeast Asia Research Collaboration with Hawaii (SEARCH), The Thai Red Cross AIDS Research Centre, Bangkok, Thailand

\section{U. Siangphoe,}

Southeast Asia Research Collaboration with Hawaii (SEARCH), The Thai Red Cross AIDS

Research Centre, Bangkok, Thailand

D. Troelstrup,

Hawaii Center for AIDS, University of Hawaii-Manoa, Honolulu, HI, USA

\section{Shikuma,}

Hawaii Center for AIDS, University of Hawaii-Manoa, Honolulu, HI, USA; Department of Medicine, University of Hawaii-Manoa, Honolulu, HI, USA; Southeast Asia Research Collaboration with Hawaii (SEARCH), The Thai Red Cross AIDS Research Centre, Bangkok, Thailand

\section{De Grutolla,}

Department of Biostatistics, Harvard School of Public Health, Boston, USA

\section{P. Sithinamsuwan,}

Division of Neurology, Department of Medicine, Phramongkutklao Hospital, Bangkok, Thailand

\section{P. Praihirunkit,}

Southeast Asia Research Collaboration with Hawaii (SEARCH), The Thai Red Cross AIDS Research Centre, Bangkok, Thailand

\section{S. Rattanamanee,}

Southeast Asia Research Collaboration with Hawaii (SEARCH), The Thai Red Cross AIDS Research Centre, Bangkok, Thailand

V. Valcour, and

(C) Journal of NeuroVirology, Inc. 2011

bshirami@hawaii.edu . 
Southeast Asia Research Collaboration with Hawaii (SEARCH), The Thai Red Cross AIDS Research Centre, Bangkok, Thailand; Memory and Aging Center, Department of Neurology, UCSF, San Francisco, USA; Division of Geriatric Medicine, Department of Medicine, UCSF, San Francisco, USA

\section{SEARCH 001.1 Study Group}

\section{Abstract}

HIV-associated neurocognitive disorders (HAND) persist despite plasma HIV RNA suppression with antiretrovirals (ARV). Sequestered reservoirs in the central nervous system and circulating monocytes are theorized to contribute to persistent brain injury. We previously demonstrated that elevated intracellular HIV DNA from circulating cells was associated with HAND in ARV-treated and ARV-naive subjects. We now report that failure to suppress intra-monocyte HIV DNA 3.5 years after initiating ARV is linked to persistent HAND and subjects with dementia are least likely to suppress intra-monocyte HIV DNA at 3.5 years. These findings suggest that antiviral strategies may need to target intra-monocyte HIV DNA.

\section{Keywords}

HIV; Dementia; HIV DNA; Neurocognitive

\section{Introduction}

The eradication of sequestered viral reservoirs has emerged as a fundamental barrier to the clearance of HIV in humans treated with combination antiretroviral therapy (CART) (Schultze et al. 2011). These reservoirs are generally thought to be quiescent but are a source for viral rebound when suppressive treatment is discontinued. Proviral HIV DNA remains detectable despite CART and the burden of virus in circulating peripheral mononuclear cells (PBMCs) is predictive of HIV disease progression and the development of AIDS (Pierson et al. 2000).

The trafficking of peripheral monocytes across the blood-brain barrier is theorized to be a mechanism of the central nervous system (CNS) HIV seeding (Gonzalez-Scarano and Martin-Garcia 2005). Animal models demonstrate migration of bone-marrow-derived monocytes from bone marrow to CNS in CD8-depleted macaques infected with simian immunodeficiency virus (Burdo et al. 2010). The burden of proviral HIV DNA in monocytes is associated with HIV-associated neurocognitive disorders (HAND) among CART-treated individuals with suppressed plasma HIV RNA and among CART-naïve subjects (Valcour et al. 2009). Mathematical modeling of CART timing suggests that the intracellular burden of proviral HIV DNA remains elevated in subjects treated during chronic infection compared to primary infection, providing a basis for a more clear understanding of longitudinal association between cognition and monocyte HIV DNA following first initiation of CART (Hocqueloux et al. 2009).

There is heightened interest in the relationship between monocyte HIV DNA and cognition given the converging interest in viral reservoir eradication for cure of HIV and recent knowledge that the prevalence of cognitive disorders has not been altered by availability of CART (Clifford 2008). We hypothesize that incompletely treated circulating viral reservoirs in monocytes increases CNS trafficking of virus, CNS immune activation and on-going synapto-dendritic injury (Ellis et al. 2007). 
This study was designed to demonstrate the long-term relationship between HIV DNA and cognition in subjects initiating first-line CART during advanced HIV disease. Our findings demonstrated selective failure to suppress HIV DNA in circulating monocytes among subjects with dementia prior to initiating CART and a persistent association between monocyte HIV DNA levels and cognition to at least 3.5 years after CART initiation, linking pre-CART HIV DNA to long-term cognitive impairment.

\section{Methods}

\section{Participant selection}

SEARCH 001 initially enrolled 30 HIV + subjects meeting Thai Ministry of Public Health guidelines for initiation of therapy (CD4 count less than 200 cells $/ \mathrm{mm}^{3}$ or symptomatic disease) and naive to CART (Valcour et al. 2007). Patients were started on CART with GPOvir ${ }^{\mathrm{TM}}$, the combination antiretroviral regimen (stavudine, lamivudine, and nevirapine) produced by the Thai Government Pharmaceutical Organization (Anekthananon et al. 2004). We enrolled 15 individuals with dementia based on the study neurologist's evaluation and use of serological, imaging, and cerebrospinal fluid evaluation to exclude opportunistic infection, if indicated. We then enrolled $15 \mathrm{HIV}+$ individuals without dementia with group matching to ensure similar age, education, gender, and CD4 lymphocyte counts between the two groups. Viral subtype for all subjects was CRF 01_AE. Baseline and 48-week results were previously reported (Valcour et al. 2010, 2007). All enrolled individuals were invited to participate in this IRB-approved 3-year extension study where they were followed every 6 months to 3.5 years.

\section{Clinical assessments}

All individuals completed longitudinal neurological examinations and neuropsychological testing. HIV-associated dementia (HAD) was clinically determined by a trained neurologist (PS) using standard-of-care assessments in Thailand, which included a comprehensive neurologic examination, simple bedside cognitive testing including the International HIV Dementia Scale, patient and proxy reporting of symptoms, brain MRI, and lumbar puncture when clinically indicated (Valcour et al. 2009). We calculated neuropsychological $z$-scores for each test using standard methodology compared to over 480 age- and education-matched Thai normative subjects. As previously described, we defined a composite neuropsychological testing summary variable (NPZ-comp) consisting of the RAVLT-total of trials 1-5 (learning efficiency), digit symbol modalities task (psychomotor speed), and timed gait (motor speed) (Valcour et al. 2009). Validation of the HAD diagnosis was performed by a consensus panel consisting of an HIV neurologist, an HIV neuropsychologist, and the principal investigator of the cohort (VV) (Valcour et al. 2009). Case summaries were prepared including individual raw neuropsychological scores plotted over three box plot distributions of seronegative control subjects, non-HAD individuals, and HAD individuals. Consensus diagnoses (HAD vs non-HAD) were determined in a blinded fashion using American Academy of Neurology 1991 criteria. Lymphocyte subsets were determined at a standard clinical reference laboratory in Thailand. Plasma HIV RNA levels were completed using the Roche ultrasensitive assay (v1.5, Roche Diagnostics, Branchburg, NJ).

\section{Isolating cellular subsets and HIV DNA quantification}

Cell separation and HIV DNA assay-Frozen PBMCs from patients for each year $\left(12 \times 10^{6}\right.$ cells) were treated with Accumax (Innovative Cell Technologies, San Diego, CA) and filtered with a 30- $\mu \mathrm{m}$ Celltrics filter (Partec, Deutschland, Germany). An aliquot of the filtered PBMC was set aside (for DNA extraction) and the remainder was separated into $\mathrm{CD} 14^{+}$monocyte and $\mathrm{CD} 14^{-}$non-monocyte populations as per manufacturer's recommendations (Human Monocyte Enrichment Kit without CD16 Depletion, StemCell 
Technologies, Vancouver, BC). DNA was extracted from the cell fractions $\left(\mathrm{CD} 14^{-}, \mathrm{CD} 14^{+}\right.$, and PBMC) using the QIAamp DNA Micro Extraction kit (Qiagen, Valencia, CA) with the DNA quality assessed using the ND-1000 spectrophotometer (NanoDrop Technologies; Wilmington, DE).

The PBMC and PBMC subset DNA were assessed for HIV DNA copy numbers by an initial pre-amplification procedure as previously described (Kusao et al. 2012). The primers used in the PCR were: HIV AE (forward 5'-GGA GCC ACC CCA CAA GAT TTA-3'; reverse 5'-GAA TAG GCC CTG CAT GTA CTG G-3') and $\beta$-globin (forward 5'-AGG GCC TCA CCA CCA ACT TC; reverse 5'-TCA CTA GCA ACC TCA AAC AGA CAC C-3') followed by multiplex PCR primers: VIC-labeled HIV gag (5'-ACC ATC AAT GAG GAA GCT GCA GAA TGG GA-3') and FAM-labeled $\beta$-globin (5'-CTC CTG AGG AGA AGT CTG CCG TTA CTG CC-3'). The copy numbers of each sample gene were analyzed against the standard curves and the HIV DNA copy number per $1 \times 10^{6}$ cells determined.

Statistical considerations-The HIV DNA data were log-transformed then plotted longitudinally to display patterns over 3.5 years. To determine the relationship between NPZ-comp and log HIV DNA for each time-point, Spearman partial correlations were computed. These partial correlations were controlled for log plasma HIV RNA at the same period of the log HIV DNA. Fisher's exact and Mann-Whitney $U$ test were used to compare categorical and continuous data between the two groups. Tests were conducted with an alpha level of 0.05. Statistical calculations were performed by SAS 9.2 (SAS Institute, Cary, NC, USA). A protocol change for PBMC isolation occurred between 3.5 and 4 years at our Thai site and resulted in unreliable HIV DNA measures from all specimens, which were subsequently censored from our analyses.

\section{Results}

As a group, the dementia cases had higher PBMC HIV DNA at baseline and this difference persisted to 1 year (Valcour et al. 2010). During the initial study period, one HAD subject died and one HAD subject was lost to follow-up. Nineteen HIV+ subjects chose to participate in the extension study (nine HAD and ten non-HAD). During this period, one additional HAD subject died resulting in 18 cases completing the 3.5-year follow-up timepoint. Among these 18 cases, the median age at baseline was 35.8 years and the median preCART CD4 count was 27 cells $/ \mathrm{mm}^{3}$ and there were no differences between the HAD and non-HAD groups. Most (15/18) identified heterosexual intercourse as the risk for HIV. As previously described, the groups differed by baseline HIV DNA levels. Among the 18 analyzed in this dataset, their pre- (baseline) median (interquartile range (IQR)) intramonocyte HIV DNA levels were 9.43 (9.28-10.59) and 2.91 (2.31-3.97) copies/106 cells for HAD and non-HAD groups, respectively $(p=0.003)$.

At 3.5 years after CART initiation, all non-HAD subjects had undetectable monocyte HIV DNA ( $<10$ copies $/ 10^{6}$ cells) compared to only four of eight HAD cases $(p=0.01)$. In contrast, there were no differences in HIV DNA levels from the monocyte-depleted PBMC fraction (Fig. 1).

Among the 18 subjects, the HAD and non-HAD groups differed on cognitive testing at baseline and at all subsequent visits throughout the longitudinal follow-up. The median (IQR) NPZ-comp score at year 3.5 was -0.07 ( -0.6 to 0.23$)$ and 0.68 (0.19 to 1.06$)$ for the $\mathrm{HAD}$ and non-HAD groups, respectively $(p=0.030)$. The partial Spearman correlation between monocyte HIV DNA and NPZ-comp score after controlling for HIV RNA levels was also significant at 3.5 years $(r=-0.580, p=0.02)$. 


\section{Discussion}

This study identified a long-term relationship between HIV DNA and cognition in subjects initiating CART with advanced disease. The data suggested that a select group of individuals initially diagnosed with HAD failed to suppress HIV DNA in circulating monocytes and this persisted for at least 3.5 years after initiation of CART. As a viral reservoir and based on existing theories of HIV neuropathogenesis, these monocytes may traffic to the CNS leading to immune activation and CNS viral particle shedding which could contribute to on-going synapto-dendritic injury (Ellis et al. 2007). These monocyte reservoirs could be established early in HIV infection leading to an emerging premise that treatment initiated during chronic HIV infection may fail to halt the injurious CNS events initiated during acute HIV infection (Denning 1988). Thus, the prevalence of cognitive disorders that continues to be described in the CART era may be partially explained by monocyte trafficking to the CNS with continued presence of intracellular HIV DNA. The monocyte HIV reservoirs are established early in HIV infection and current treatment strategies fail to target these cells potentially leading to continued insult to the brain (Denning 1988).

The current study enrolled in a geographic region where combination recombinant form CRF 01 AE was predominant. Previous data from a cohort in Hawaii where HIV Clade B is predominant demonstrated higher monocyte HIV DNA levels in individuals with HAD compared to those with non-HAD (Kusao et al. 2012). We have also reported that HIV DNA is linked to cognition in well-treated chronic HIV infection (Shiramizu et al. 2005). More recently, higher HIV Clade B DNA levels in activated monocyte were found in patients with persistent neurocognitive deficits longitudinally (Kusao et al. 2012), which is consistent with the current study demonstrating the longitudinal association between failure to clear HIV DNA and persistent impairment. We hypothesize that the continual presence of HIV DNA in circulating monocytes provided a persistent barrage of HIV-infected monocytes trafficking to the brain. In the work presented here, there appeared to be a subset of HIVinfected individuals who have low level monocyte HIV DNA prior to CART and these individuals initially presented and remained without cognitive impairment while on CART. Monocytes isolated from individuals with normal cognition not only had lower HIV DNA copy numbers but the monocytes had a less pro-inflammatory phenotype based on the levels of cytokines secreted by the cells (Kusao et al. 2012). One paradigm that is consistent with the current hypothesis is that the patients' immune systems represent a baseline spectrum of inflammatory processes. Individuals who are in a chronic inflammatory state would have more circulating activated monocytes, which would be prone to HIV infection, thus more of these infected monocytes could traffic to the brain setting these individuals up for increased risk of HAD (Ellery et al. 2007; Munsaka et al. 2009). In this theory, subjects with a lower inflammatory state would have less monocytes activated and lower HIV DNA in monocytes trafficking to the CNS. An alternative theory is that the HIV DNA infection itself drives the activation. This would suggest distinct mechanisms with cellular infection as the pivotal driver.

Our data provided evidence demonstrating a selective failure to eradicate HIV DNA in circulating monocytes among subjects with dementia. However, the study was limited by the small number of individuals in the cohort. Validation of the results will be required in a larger cohort over a longer period in order to move forward in designing treatment strategies targeting circulating monocytes in the future. 


\section{Acknowledgments}

The authors thank the patients and staff from the Phramongkutklao Medical Center, SEARCH. and the Hawaii Center for AIDS; AFRIMS, Bethesda, and UCSF. Supported in part by NS061696, MH072388, K23AG032872, RR026136, and NS053345.

The SEARCH 001 protocol team: Phramongkutklao Hospital: Samart Nidhinandana, Sataporn Thitivichianlert, Thippawan Chuenchitra, Suchitra Sukwit, Suwicha Chitpatima; SEARCH: Wichitra Apateerapong, Benjawan Boonchokchai, George Watt, Duanghathai Suttichom, Nitiya Chomchey; Hawaii Center for AIDS: Van Nicholas Velasco; AFRIMS: Rapee Trichavaroj.

\section{References}

Anekthananon T, Ratanasuwan W, Techasathit W, Sonjai A, Suwanagool S. Safety and efficacy of a simplified fixed-dose combination of stavudine, lamivudine and nevirapine (GPO-VIR) for the treatment of advanced HIV-infected patients: a 24-week study. J Med Assos Thail Suppl. 2004; 87:760-767.

Burdo TH, Soulas C, Orzechowski K, Button J, Krishnan A, Sugimoto C, Alvarez X, Kuroda MJ, Williams KC. Increased monocyte turnover from bone marrow correlates with severity of SIVencephalitis and CD163 levels in plasma. PLoS Pathog. 2010; 6:e1000842. [PubMed: 20419144]

Clifford DB. HIV-associated neurocognitive disease continues in the antiretroviral era. Top HIV Med. 2008; 16:94-98. [PubMed: 18591717]

Denning DW. The neurological features of acute HIV infection. Biomed Pharmacother. 1988; 42:1114. [PubMed: 3044455]

Ellery PJ, Tippett E, Chiu YL, Paukovics G, Cameron PU, Solomon A, Lewin SR, Gorry PR, Jaworowski A, Greene WC, Sonza S, Crowe SM. The CD16 ${ }^{+}$monocyte subset is more permissive to infection and preferentially harbors HIV-1 in vivo. J Immunol. 2007; 178:6581-6589. [PubMed: 17475889]

Ellis R, Langford D, Masliah E. HIV and antiretroviral therapy in the brain: neuronal injury and repair. Nat Rev Neurosci. 2007; 8:33-44. [PubMed: 17180161]

Gonzalez-Scarano F, Martin-Garcia J. The neuropathogenesis of AIDS. Nat Rev Immunol. 2005; 5:69-81. [PubMed: 15630430]

Hocqueloux, L.; Avettand-Fenoel, V.; Jacquot, S.; Prazuck, T.; melard, A.; Viard, J.; Le Moat, G.; Rouzioux, C. Better HIV DNA depletion and CD4 restoration with HAART initiated at the time of primary HIV infection than with HAART started during chronic HIV infection; 16th Conference of Retroviruses and Opportunistic Infections; Montreal, CA. 2009; Abstract 515

Kusao I, Shiramizu B, Liang C-Y, Grove J, Agsalda M, Troelstrup D, Velasco V-N, Marshall A, Whitenack N, Shikuma C, Valcour V. Cognitive Performance Related to HIV-1-Infected Monocytes. J Neuropsych Clin Neurosc. 2012 In press.

Munsaka SM, Agsalda M, Troelstrup D, Hua N, Yua Q, Shiramizu B. Characteristics of activated monocyte phenotype support R5-tropic human immunodeficiency virus. Immunology and Immunogenetics Insights. 2009; 1:15-20. [PubMed: 19997535]

Pierson T, McArthur J, Siliciano RF. Reservoirs for HIV-1: mechanisms for viral persistence in the presence of antiviral immune responses and antiretroviral therapy. Annu Rev Immunol. 2000; 18:665-708. [PubMed: 10837072]

Schulze J, zur Wiesch J, van Lunzen J. Hide and seek...can we eradicate HIV by treatment intensification? Infect Dis. 2011; 203:894-897.

Shiramizu B, Gartner S, Williams A, Shikuma C, Ratto-Kim S, Watters M, Aguon J, Valcour V. Circulating proviral HIV DNA and HIV-associated dementia. Aids. 2005; 19:45-52. [PubMed: 15627032]

Valcour VG, Sithinamsuwan P, Nidhinandana S, Thitivichianlert S, Ratto-Kim S, Apateerapong W, Shiramizu BT, Desouza MS, Chitpatima ST, Watt G, Chuenchitra T, Robertson KR, Paul RH, McArthur JC, Kim JH, Shikuma CM. Neuropsychological abnormalities in patients with dementia in CRF 01_AE HIV-1 infection. Neurology. 2007; 68:525-527. [PubMed: 17296919] 
Valcour VG, Shiramizu BT, Sithinamsuwan P, Nidhinandana S, Ratto-Kim S, Ananworanich J, Siangphoe U, Kim JH, de Souza M, Degruttola V, Paul RH, Shikuma CM. HIV DNA and cognition in a Thai longitudinal HAART initiation cohort: the SEARCH 001 Cohort Study. Neurology. 2009; 72:992-998. [PubMed: 19289739]

Valcour VG, Shiramizu BT, Shikuma CM. HIV DNA in circulating monocytes as a mechanism to dementia and other HIV complications. J Leukoc Biol. 2010; 87:621-626. [PubMed: 20130221] 

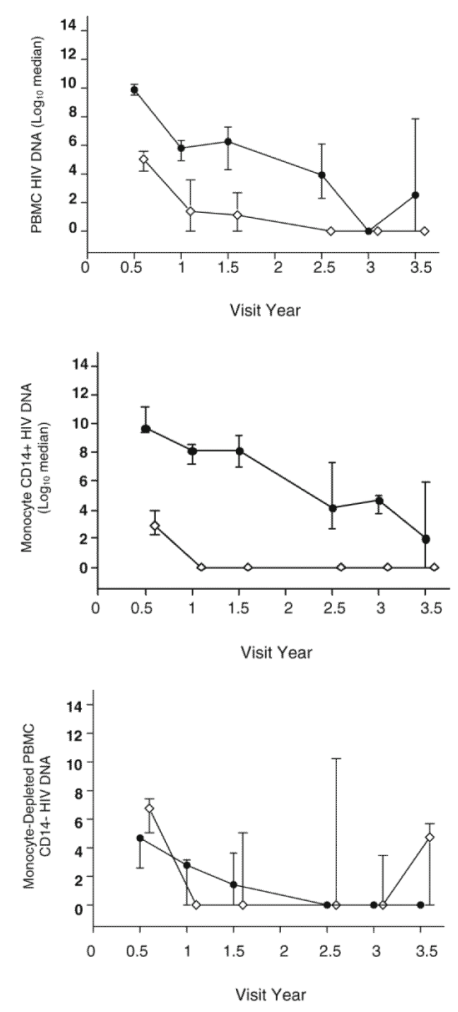

Fig. 1.

HIV DNA ( $\log _{10}$ median with inter-quartile range) in peripheral mononuclear cells (PBMC, top; monocytes, middle; and monocyte-depleted PBMC, bottom) comparing dementia cases (black circle) to non-dementia cases (white circle). Some CART-naïve individuals with dementia at baseline were unable to suppress monocyte HIV DNA after 3.5 years on CART resulting in a higher median HIV DNA copies in the dementia group 